\title{
Joint Computational and Experimental Investigations on the Synthesis and Properties of Hantzsch-type Compounds An Overview
}

*Department of Chemistry, Faculty of Physics \& Chemistry, Alzahra University, Tehran, Iran

\begin{abstract}
In this review, we try to highlight the significance, mechanism propositions, computational and experimental assessments of Hantzsch dihydropyridine (DHPs) which readily oxidized to the corresponding pyridines as one of the most important aromatic heterocycles. We also try to give an overview to its ability in transfer hydrogenation, acting as hydride donors from computational and experimental points of view. Our survey is also extended to computational assessments on the structural and biological properties of Hantzsch DHPs.
\end{abstract}

Keywords: Hantzsch synthesis; asymmetric transfer hydrogenation; biological properties; calcium channel blockers; computational chemistry; dihydropyridines 